\title{
Analysis of mitochondrial DNA in Leber's hereditary optic neuropathy
}

\author{
J Poulton, M E Deadman, J Bronte-Stewart, W S Foulds, R M Gardiner
}

\begin{abstract}
Twenty-eight patients from 25 maternal lineages with Leber's hereditary optic neuropathy (LHON) were investigated by restriction enzyme analysis for the presence or absence of the point mutation described by Wallace et al. The mutation was identified in 18 of $25(72 \%)$ families with LHON. This provides further evidence that this mutation is present in the majority of patients with LHON. In 19 of these families with LHON, additional analysis using sequencing, oligonucleotide probing, and competitive oligonucleotide priming of PCR products was performed. In 14 cases with the site loss the point mutation was present, and five without the site loss had the wild type sequence in this region.
\end{abstract}

The maternal pattern of inheritance in Leber's hereditary optic neuropathy (LHON) ${ }^{1}$ led to suggestions that it might be caused by a mutation in mitochondrial DNA (mtDNA), because mtDNA is exclusively maternally inherited. Early restriction enzyme analysis of blood $\mathrm{mtDNA}$ showed neither a major rearrangement, nor an association with a particular mtDNA type. ${ }^{2}{ }^{3}$ However, a more detailed study identified a point mutation in mtDNA. ${ }^{1}$ Indirect evidence suggests that it is causative, but no definite mechanism has yet been identified. Not only is the

Department of Paediatrics, University of Oxford, John Radcliffe Hospital, Headington, Oxford.

J Poulton, M E Deadman

Tennent Institute of Ophthalmology, University of Glasgow, Church Street, Glasgow.

J Bronte-Stewart, W S Foulds

Department of Paediatrics, University College and Middlesex School of Medicine, University College London, London.

R M Gardiner

Correspondence to Dr Poulton, Genetics Laboratory, Department of Biochemistry, University of Oxford, South Parks Road, Oxford OX1 3QU.

Received for publication 5 February 1991.

Revised version accepted for publication 14 May 1991. mutation present in the majority of LHON families ${ }^{4-6}$ but it has been confirmed in several different ethnic groups $\mathrm{s}^{6-8}$ and comparison of mtDNA typing suggests that it occurred independently at least twice in the past 40 to 80000 years. ${ }^{8}$ This mutation is an $\mathrm{A}$ to $G$ transition at base pair number 11778 , and results in the replacement of a conserved arginine residue with a histidine. It can be detected by Southern hybridisation because it causes an $S f a \mathrm{NI}$ site loss. While some families appear to be homoplasmic for this defect (a single population of mtDNA in each subject), others are heteroplasmic (two or more populations of mtDNA in the same subject). Severity appears to correlate with the dosage of abnormal $\mathrm{mtDNAs}{ }^{59}$

We have studied 28 patients with LHON and 12 controls who included three patients with dominantly inherited optic neuropathy and nine normally sighted subjects. Southern hybridisation, sequence analysis, oligonucleotide probing, and competitive oligonucleotide priming (COP) of PCR products of blood DNA were used to detect the presence of this point mutation. These last three methods were used in addition to Southern hybridisation, to obtain more information than is apparent from the loss of a restriction enzyme site. Sequence analysis was performed on blood from five LHON patients in whom the $S f a \mathrm{NI}$ site was intact in order to ascertain whether there was an alteration in the first base position of the arginine codon which would not intersect the enzyme's recognition sequence. Oligonucleotide probing was performed in order to confirm that the site loss was the result of the same base change in all patients and to confirm the sequence data. COP was used in an attempt to investigate heteroplasmy, as it may be more sensitive at detecting minority populations than the other methods.

\section{Methods \\ PATIENTS}

These were diagnosed at the Tennent Institute of Ophthalmology, Glasgow, by Dr Bronte-Stewart and Professor Foulds (22 cases), the Birmingham and Midland Eye Hospital (two cases), The Hospital for Sick Children, Great Ormond Street, London (three cases), and at the Oxford Eye Hospital by 
Professor Bron (one case). Clinical details are summarised in table 1. Blood was obtained from affected subjects (28 cases) and four unaffected relatives. Cases 28 and 29 (both affected) were the parents of case 27. The other patients with LHON included two sib pairs (cases 23 and 24, and 25 and 26). There was a father-son pair among the patients with dominantly inherited optic atrophy (cases 7 and 8 ).

Diagnostic criteria for $\mathrm{LHON}^{10}$ included acute painless loss of vision becoming progressively severe over a period of days, and becoming bilateral usually within weeks, but not longer than nine months, accompanied by optic disc swelling and hyperaemia at onset and optic atrophy subsequently together with a dense central on centrocaecal scotoma in the field of vision or an acquired red/green dyschromatopsia. An additional feature in most cases was glial sheathing of retinal vessels in the vicinity of the optic nerve head. As there is no absolute diagnostic feature, only patients considered typical or with a clear family history were included. In contrast, patients with dominantly inherited optic atrophy (dominant OA) had moderate or mild non-progressive visual impairment and an autosomal dominant pattern of inheritance.

All families (including 19 of the patients with LHON) were investigated using Southern hybridisation, oligonucleotide probing, and competitive oligonucleotide priming, except for nine LHON patients from six maternal lineages in whom only restriction enzyme analysis was performed. Sequence analysis was carried out in five of the LHON patients in whom there was no SfaNI site loss.

\section{SOUTHERN HYBRIDISATION}

Blood DNA was digested with SfaNI, electrophoresed on $1.5 \%$ gels, Southern blotted, and probed with a human mtDNA probe in bacteriophage $\mathrm{m} 13$ corresponding either to base pairs 10254 to 11922 (probe 11) or to base pairs 11680 to 12570 (probe 12) (both courtesy of Professor G Attardi and Dr M

Table 1 Patient details.

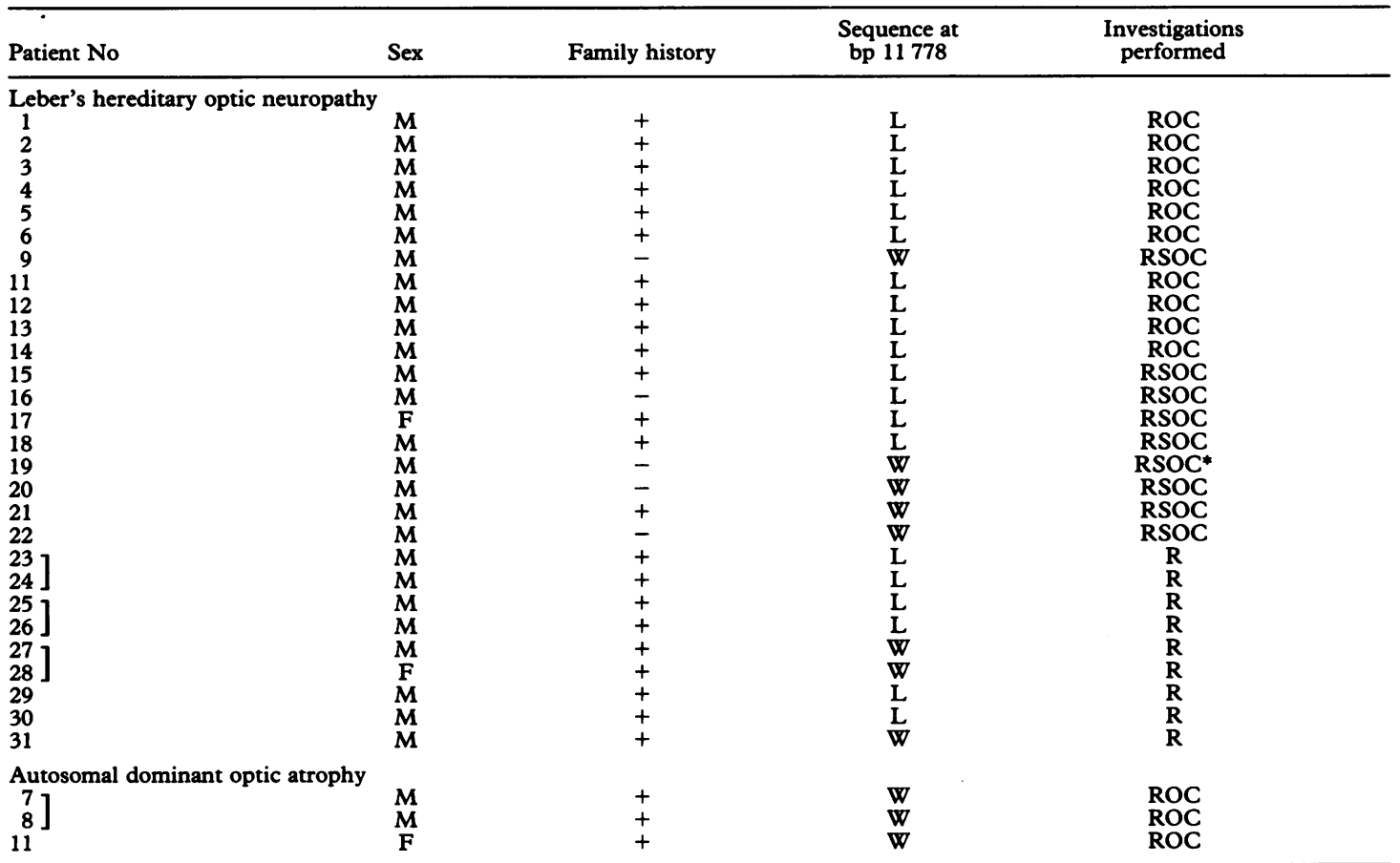

$+=$ other affected family members consistent with diagnosis.

$-=$ sporadic.

$\mathrm{L}=11778$ mutation present.

$\mathrm{W}=$ normal sequence at bp 11778 .

] = same maternal lineage (see text).

$\mathbf{R}=$ restriction enzyme analysis.

$\mathrm{S}=$ sequence analysis.

$\mathrm{O}=$ oligonucleotide probing.

$\mathrm{C}=$ competitive oligonucleotide priming.

${ }^{*} R$ and $S$ on proband and asymptomatic mother, $O$ and $C$ on mother only. 
King). At least two autoradiographs were obtained: overnight and overexposed (up to nine days) in order to detect heteroplasmy.

\section{POLYMERASE CHAIN REACTION}

This was carried out in a computer operated thermal cycler with thermostable $T a q$ polymerase (Cetus) 1 unit per 50 reaction along with buffers and mineral oil as recommended by the manufacturer. Concentrations of magnesium and primers and annealing temperature were optimised for each primer pair; $100 \mathrm{ng}$ of template was used unless otherwise stated.

\section{SEQUENCE ANALYSIS}

PCR was used to amplify a $231 \mathrm{bp}$ region of MTND4 (with primers ND4/6 and ND4/7 corresponding to base pairs 11632 to 11651 and complementary to 11843 to 11862 respectively) from patients and controls for this and the remaining analyses. In two patients (nos 20 and 21) the PCR product was cloned into phosphatased SmaI cut bacteriophage M13 after 'filling in' using Klenov and kinasing as described by Poulton et al. ${ }^{11}$ Direct sequencing of PCR products was also performed in these and the other two LHON patients and one mother with an intact $S f a \mathrm{NI}$ site (patients 9,19 , and 22) and patients 15 to 18 using the unbalanced priming method. ${ }^{12}$ Primers were either ND4/1 and ND4/3 (corresponding to bp 11090 to 11109 and complementary to 12172 to 12191 respectively) or ND4/6 and ND4/7 followed by unbalanced priming with ND4/6 and 7 (an excess of ND4/6 followed by sequencing with ND4/7 or an excess of ND4/7 followed by sequencing with ND4/6). Primer concentrations were $500 \mathrm{nmol} / 1$ and $5 \mathrm{nmol} / 1$, magnesium concentration $1 \mathrm{mmol} / \mathrm{l}$.

\section{OLIGONUCLEOTIDE PROBING}

Approximately $200 \mathrm{ng}$ of the same $231 \mathrm{bp}$ PCR product described above was dot blotted. This filter was probed with allele specific oligonucleotides which had been radiolabelled with gamma 32P-
$\mathrm{ATP}^{13}$ (ND4/W and ND4/L, corresponding to the wild type and the mutated sequence complementary to base pairs 11769 to 11784 ).

\section{COMPETITIVE OLIGONUCLEOTIDE PRIMING}

Competitive oligonucleotide priming is similar in principle to oligonucleotide probing, only it uses PCR to detect hybridisation. ${ }^{14}$ Both allele specific primers are present in the reaction along with an appropriately placed oligonucleotide for priming synthesis of the complementary strand. The alternative primers compete with each other but the exact match primes about 100 times more often. This can be shown by labelling one or other of the primers and investigating incorporation of radioactivity into the product by electrophoresis (fig 1). Primers ND4/ $\mathrm{W}$ and $\mathrm{ND} 4 / \mathrm{L}$ were end labelled using $\mathrm{T} 7$ polynucleotide kinase with gamma 32P-ATP, and PCR was performed along with primer ND4/6 using the following cycles: denaturation $93^{\circ} \mathrm{C}$ for 60 seconds, annealing $43.5^{\circ} \mathrm{C}$ and $46.5^{\circ} \mathrm{C}$ respectively for 60 seconds, extending $55^{\circ} \mathrm{C}$ for 60 seconds for 20 cycles. Magnesium concentration was $4 \mathrm{mmol} / \mathrm{l}$ and primer concentrations $500 \mathrm{nmol} / \mathrm{l}$.

\section{Results}

SfaNI SITE LOSS AT BP 11778 IN 28 PATIENTS

The mutation was identified in 18 of $25(72 \%)$ families with LHON (tables 1 and 2). Seventeen out of 18 of these were familial, whereas four of the seven cases who did not have the mutation were sporadic. In one of these seven cases, the diagnosis

Table 2 Incidence of point mutation at bp 11778 and family history in 25 families with LHON.

\begin{tabular}{|c|c|c|c|}
\hline & Familial & Sporadic & Total \\
\hline & No $(\%)$ & No $(\%)$ & No $(\%)$ \\
\hline $\begin{array}{l}\text { LHON: with } 11778 \text { mutation } \\
\text { Wild type at } 11778 \\
\text { Total }\end{array}$ & $\begin{array}{rl}17 & 85 \\
3 & 15 \\
20 & \end{array}$ & $\begin{array}{l}120 \\
480 \\
5\end{array}$ & $\begin{array}{rl}18 & 72 \\
728 \\
25 & 100\end{array}$ \\
\hline
\end{tabular}

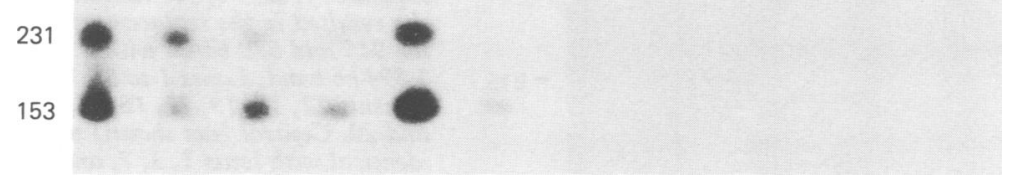

Figure 1 Competitive oligonucleotide priming. Lanes 1 and 7: patient 15. Lanes 2 and 8, 3 and 9: controls. Lanes 4 and 10: patient 21. Lanes 5 and 11: patient 20. Lanes 6 and 12: patient 19 (loaded in this order to minimise confusion from overflow from one well to the next). The oligonucleotide specific for the 11778 mutation was end labelled in lanes 1 and 8 to 12, the wild type oligonucleotide was end labelled in lanes 2 to 7. 
was in some doubt, as microangiopathy was only observed in a small area above one disc. Of the remaining three, all were considered typical, although two patients were not personally examined at presentation. There appeared to be two independent maternal lineages transmitting the LHON phenotype in the family comprising a proband with affected parents: the father carried the mutation but the mother and proband did not. Identical results were obtained where more than one member of a maternal lineage were investigated.

\section{DETAILED ANALYSIS OF 19 PATIENTS \\ Southern hybridisation}

Loss of the $S f a$ NI site resulted in the replacement of the 915 and 679 bands with a $1.594 \mathrm{~kb}$ band in 14 of 19 subjects (fig 2). No evidence of heteroplasmy was seen.

\section{Sequence analysis}

In all cases the results confirmed the data shown in table 2. Normal sequence in this region was obtained in all subjects in whom the $S f a$ NI site was intact. In particular, there were no alterations in the first base position of the arginine codon which would not interrupt the enzyme's recognition sequence.

\section{Oligonucleotide probing}

The signal was positive with either one or the other probe (fig 3), providing no evidence of heteroplasmy. Further, it confirmed that the site loss was the result of the G to A transition in the 14 of 19 LHON families (tables 1 and 2). It also confirmed that the wild type sequence was present in the remaining four LHON patients tested, one mother, and the controls.
Competitive oligonucleotide priming

This result was further confirmed using competitive oligonucleotide priming as shown in fig 1 . Radiolabelled primers were only incorporated in reactions where they exactly corresponded to the template sequence when 20 cycles were performed using annealing temperatures of $48^{\circ} \mathrm{C}$ or above. At lower temperatures (below 43.5 and $46.5^{\circ} \mathrm{C}$ for ND4/W and ND4/L respectively) non-specific priming occurred: incorporation into PCR product using cloned wild type template was similar to that obtained when the template was also contaminated with $1 \%$ of DNA from a mutant LHON product and vice versa. No evidence of heteroplasmy was obtained.

\section{Discussion}

The point mutation at bp 11778 was identified in 18 of 25 or $72 \%$ of families with LHON, but not in the three patients with dominantly inherited optic atrophy or the nine controls. This is comparable to the original study of Wallace et $a l^{4}$ (where the mutation was found in 9 of 11 or $81 \%$ of patients). This is considerably higher than the 10 of 19 found in the study of Vilkki $e t a l^{6}$ from Finland or the 4 of 8 families in the study of Holt et al. ${ }^{5}$ It is unlikely that this is because of differences in diagnostic criteria, as the proportion was similar in both certain and probable Finnish cases. They also showed that there was no recent common ancestor for 11 of their families. These authors ${ }^{6}$ suggested that more than one mtDNA mutation is able to cause the LHON phenotype. The differences between the studies therefore probably reflect different incidence of causative mutations in different maternal lineages.

The majority of the cases where the mutation was present were familial, whereas half of the cases who did not have the mutation were sporadic. There are several possible explanations for this. Firstly, there is no absolute diagnostic criterion for LHON. The absence of family history makes the diagnosis less

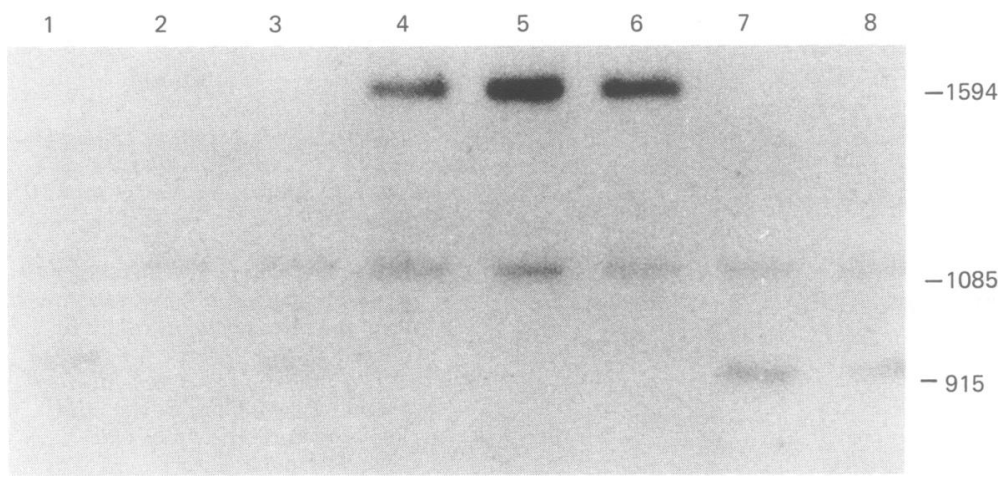

Figure 2 Blood DNA was digested with SfaNI, electrophoresed in $1.5 \%$ agarose gels, Southern blotted, and probed with regional probe 11 (corresponding to bp 10 , 254-11922 of the reference sequence $\left.{ }^{15}\right)$. Loss of the restriction site resulted in the replacement of the 915 and 679 bands with $a$ $1.594 \mathrm{~kb}$ band. Lanes 1 to 8 : patients 22, 18, 19, 17, 16, 15, 21, and 20. Control (not shown) was identical with lanes $1,3,7$, and 8. 
Wild (ND4/W)

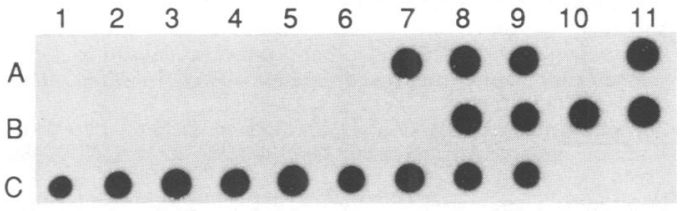

Mutated (ND4/L)

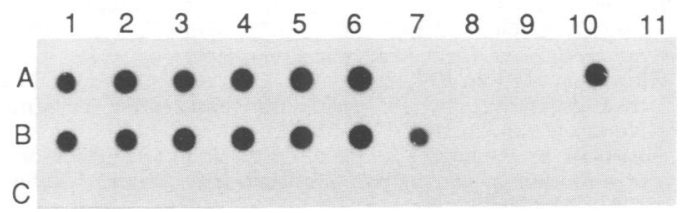

Figure 3 Dot blot filter of PCR product ND4/6-ND4/7 probed with end labelled ND4/W (or the oligonucleotide corresponding to the wild type sequence) (upper photograph) and ND4/L (or the oligonucleotide corresponding to the mutated sequence) (lower photograph). Row $A$, columns 1 to 11 : patients 1 to 11 . Row $B$, columns 1 to 11 : patients 12 to 22 . Row C, columns 1 to 9: controls.

certain in the four cases in whom the major population of mtDNA has the wild type sequence in this region: optic atrophy could be non-genetic in origin, or be caused by a nuclear rather than a mitochondrial mutation. Alternatively, they might exhibit heteroplasmy with a wide variation in genotypic ratio between tissues: there might have been a somatic mutation in the developing eye at bp 11778 which is not detectable in blood. Unfortunately, this hypothesis is difficult to test, as appropriate pathological specimens are rare because patients with LHON die from other causes. Finally, it is possible that some of the other causative mitochondrial point mutations which have been suggested actually influence survival, and hence reduce the likelihood of a family history. Further sequence analysis is in progress in the families in which the 11778 mutation was not detected. Nineteen patients and 12 controls were studied in more detail, using oligonucleotide probing, sequencing, and competitive oligonucleotide priming in addition to restriction enzyme analysis. All three methods confirmed that 14 out of the 19 patients with LHON had the expected point mutation. Sequence data on the five patients without the site loss excluded substitutions of the same amino acid arising from other mutations.

None of the methods used provided support for the observation of Holt $e t a l^{5}$ and Vilkki et al $l^{6}$ that heteroplasmy is present in a proportion of affected subjects with the 11778 mutation, and that there is a dosage effect. There are a number of possible reasons for this discrepancy. Firstly, the levels of normal mtDNAs in the group of 14 subjects may be extremely low: this group was mainly affected patients and only three asymptomatic relatives who might be expected to carry a detectable level of normal mitochondrial genomes in blood. Secondly, only patients who very clearly fulfilled the diagnostic criteria were included in this series: it is possible that if patients who were less severely affected had been included the levels of normal mtDNA might have been higher. Thirdly, none of the techniques used was especially sensitive for detecting low levels of normal mtDNAs. Southern hybridisation can detect levels down to about $5 \%$ and oligonucleotide probing is less sensitive than this. Sequence analysis of a single clone is likely to reflect the major population of mtDNA, and it would be necessary to sequence 15 clones in order to be more than $80 \%$ certain of detecting a minority population of less than $10 \%$ of $\mathrm{mtDNAs}$. Direct sequencing of PCR products has been used to detect heterozygosity of nuclear genes but, in practice, 'ghosting' prevents detection of low levels of abnormal genomes with certainty on a single run. It was anticipated that competitive oligonucleotide priming would be able to detect low levels of minority populations of mtDNA. In practice, under conditions where the minority population of mtDNA in a 99:1 mix gave a signal, there was considerable non-specific priming for both primers. The level of this non-specific background depended on total yield of PCR product. The variation in yield between different templates in the same reaction resulted in varying backgrounds and precluded the identification of heteroplasmy. This might be simply a property of the primers used, and it is likely that other techniques based on PCR would be effective.

These data provide further evidence that the mutation at base pair 11778 is present in the majority of patients with LHON. While other mutations in mitochondrial DNA may be present in the remaining patients, these have yet to be identified.

Financial support was provided by the National Fund for Research into Crippling Diseases, the Muscular Dystrophy Group, British Medical Association (Vera Down Award), the E P Abraham Trust, the Medical Research Council, the Sir Halley Stewart Trust, and the Medical Research Fund of Oxford University. We thank the patients and their families, and their physicians (Dr S Bundey, Dr J Wilson, Dr P Phelan, Professor A Bron, Professor J Newsom-Davis) for helpful cooperation, Professor G Attardi and Dr M King for allowing us to use the mitochondrial probes, $\mathrm{Dr} \mathrm{N}$ Howell for assistance, Professor A C Wilson and associates for providing primers and instruction in the use of PCR, Dr T Kocher for making the alternating temperature water bath, Kathy Shaw for typing the manuscript, 
and Professor E R Moxon for his support and encouragement.

1 Seedorff T. The inheritance of Leber's disease. Acta Ophthamol (Copenh) 1985;63:135-45.

2 Holt IJ, Miller DH, Harding AE. Mitochondrial DNA polymorphism in Leber's optic neuropathy. $\mathcal{F}$ Neurol Neurosurg Psychiatry 1988;51:1075-7.

3 Vilkki J, Savontaus M, Kalimo H, Nikoskelainen EK. Mitochondrial DNA polymorphism in Finnish families with Leber's hereditary optic neuroretinopathy. Hum Genet 1989;82:208-12.

4 Wallace DC, Singh G, Lott MT, et al. Mitochondrial DNA mutation associated with Leber's hereditary optic neuropathy. Science 1988;242:1427-30.

5 Holt IJ, Miller DH, Harding AE. Genetic heterogeneity and mitochondrial DNA heteroplasmy in Leber's hereditary optic neuropathy. $f$ Med Genet 1989;26:739-43.

6 Vilkki J, Savontaus M, Nikoskelainen EK. Genetic heterogeneity in Leber hereditary optic neuropathy revealed by a mitochondrial DNA polymorphism. Am F Hum Genet 1989;45:206-11.

7 Ozawa T, Tanaka $M$. Types and mechanisms of mitochondrial DNA mutations. International symposium on Molecular basis of neurological disorders and their treatment. (IBMI symposium no 5.) Fasano, Italy, 1989.
8 Singh G, Lott MT, Wallace DC. A mitochondrial DNA mutation as a cause of Leber's hereditary optic neuropathy. N Engl f Med 1989;320:1300-5.

9 Vilkki J, Savontaus M, Nikoskelainen EK. Segregation of mitochondrial genomes in a heteroplasmic lineage in Leber hereditary optic neuroretinopathy. Am 7 Hum Genet 1990;47:95-100.

10 Nikoskelainen E. The clinical findings in Leber's hereditary optic neuropathy. Trans Ophthalmol Soc UK 1985; 104:845-52.

11 Poulton J, Deadman ME, Turnbull DM, Lake B, Gardiner RM. Detection of mitochondrial DNA deletions in blood using the polymerase chain reaction: non-invasive diagnosis of mitochondrial myopathy. Clin Genet 1991;39:33-8.

12 Gyllensten U. Direct sequencing of in vitro amplified DNA. In: Erlich HA, ed. PCR technology: principles and applications for DNA amplification. New York: Stockton Press, 1989.

13 Kogan SC, Gitschier J. Genetic prediction of haemophilia. In: Innis MA, Gelfand DH, Sninsky JJ, White TJ, eds. PCR protocols. New York: Academic Press, 1990.

14 Gibbs RA, Nguyen PN, Caskey CT. Detection of single DNA base differences by competitive oligonucleotide priming. Nucleic Acids Res 1989;17:2438-48.

15 Anderson S, Bankier AT, Barrell BG, et al. Sequence and organisation of the human mitochondrial genome. Nature 1981;290:457-65. 